\title{
Progress on a Rayleigh Scattering Mass Flux Measurement Technique
}

\author{
Amy F. Mielke-Fagan ${ }^{*}$, Michelle M. Clem ${ }^{\dagger}$ \\ NASA Glenn Research Center, Cleveland, Ohio, 44135 \\ Kristie A. Elam ${ }^{\ddagger}$ \\ Jacobs Sverdrup, Cleveland, OH 44135 \\ and \\ Stefanie M. Hirt ${ }^{\S}$ \\ NASA Glenn Research Center, Cleveland, Ohio, 44135
}

\begin{abstract}
[Abstract] A Rayleigh scattering diagnostic has been developed to provide mass flux measurements in wind tunnel flows. Spectroscopic molecular Rayleigh scattering is an established flow diagnostic tool that has the ability to provide simultaneous density and velocity measurements in gaseous flows. Rayleigh scattered light from a focused 10 Watt continuous-wave laser beam is collected and fiber-optically transmitted to a solid FabryPerot etalon for spectral analysis. The circular interference pattern that contains the spectral information that is needed to determine the flow properties is imaged onto a CCD detector. Baseline measurements of density and velocity in the test section of the $15 \mathrm{~cm} \times 15$ cm Supersonic Wind Tunnel at NASA Glenn Research Center are presented as well as velocity measurements within a supersonic combustion ramjet engine isolator model installed in the tunnel test section.
\end{abstract}

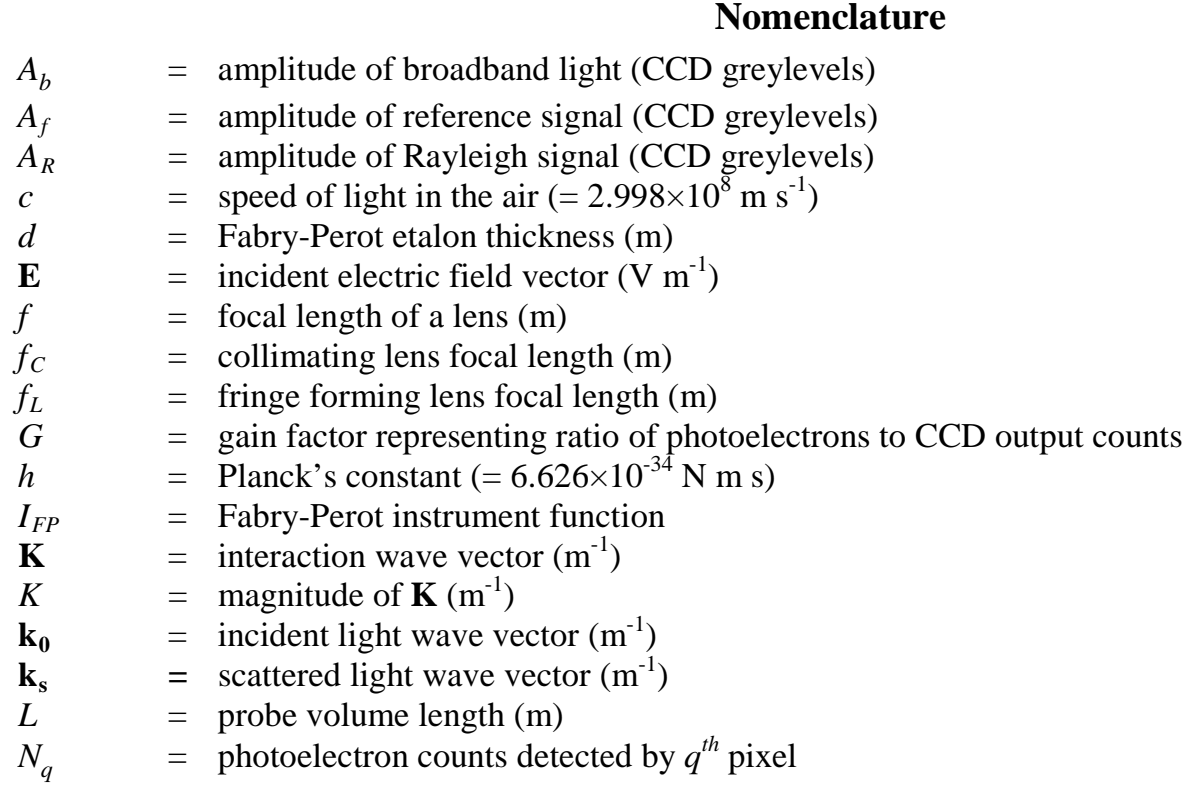

\footnotetext{
* Research Engineer, Optical Instrumentation/NDE Branch, 21000 Brookpark Rd/M.S. 77-1, AIAA Senior Member

${ }^{\dagger}$ Research Engineer, Optical Instrumentation and NDE Branch, 21000 Brookpark Rd/M.S. 77-1

‡ Optics Technician, 21000 Brookpark Rd/M.S. 77-3

$\S$ Aerospace Engineer, Inlet and Nozzle Branch, 21000 Brookpark Rd/M.S. 5-12, AIAA Senior Member
} 


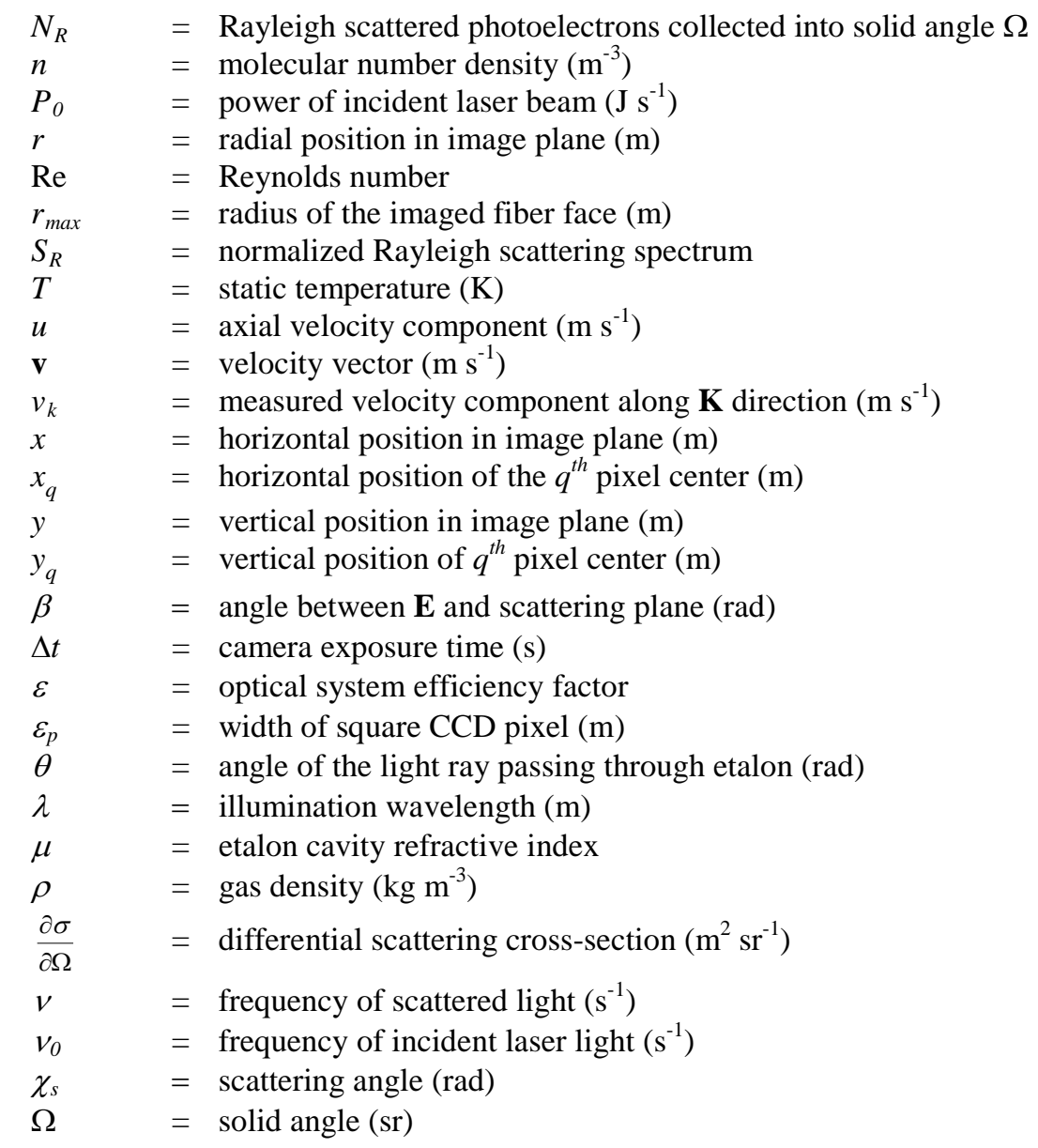

\section{Introduction}

R AYLEIGH scattering, which as has been used to obtain measurements in various flow environments, is the elastic scattering of light from molecules where the signal strength, Doppler frequency shift, and spectral linewidth of the scattered light provide measurements of density, velocity, and temperature, respectively ${ }^{1}$. Since no energy exchange takes place between the photons and molecules, any laser frequency is applicable, although shorter wavelengths are typically desired since the scattering strength scales by $\lambda^{-4}$. Typical Rayleigh linewidths are on the order of $1 \mathrm{GHz}$ and Doppler frequency shifts are on the order of $1-2 \mathrm{MHz} / \mathrm{m} / \mathrm{s}$; hence a very narrow linewidth laser ( $\sim \mathrm{MHz})$ and an extremely high resolution filter are required to resolve the spectrum. A Fabry-Perot (FP) interferometer or etalon is a high resolution filter based on interference phenomena which is used to resolve the spectrum of the light in this work. A FP etalon consists of two parallel planar reflective plates or surfaces and is typically used in the imaging mode (constant spacing between reflective surfaces) for interferometric Rayleigh scattering $^{2}$. An etalon may be air-spaced or consist of a solid transparent optical material (a solid fused silica etalon is used in this work). When light is imaged through the etalon an interference pattern results which is a function of the spectrum of the light convolved with the instrument function of the Fabry-Perot. The Fabry-Perot instrument function is the well-known Airy function. A model function utilizing a Rayleigh spectrum model and the Airy function is fit to the recorded interference pattern to provide flow measurements.

Rayleigh measurements have been performed in wind tunnels previously by various research groups ${ }^{3,4}$. Bivolaru et al. $^{3}$ has made velocity measurements in a combustion-heated supersonic jet facility. Seasholtz et al. ${ }^{4}$ made velocity measurements in a supersonic wind tunnel where they used an iodine absorption filter to remove unwanted stray laser light. Our research group at NASA Glenn Research Center (GRC) has developed several fiber-coupled point-wise Rayleigh scattering measurement systems that are capable of acquiring data at high sampling rates using high quantum efficiency detectors ${ }^{5-7}$. In most of our previous work an air-spaced FP interferometer was used to measure the Rayleigh spectrum. In these studies the FP interferometer had to be remotely located from the harsh facility environments and the Rayleigh signal fiber-coupled to the interferometer since an air-spaced etalon does not 
have a rigid fixed spacing and vibrations can change the plate spacing and corrupt the spectral measurements. In more recent work a rigid solid fused-silica FP etalon has been used to allow spectral analysis to take place in close proximity to the harsh facilities. A solid etalon was used to implement an approach to provide spatially-resolved Rayleigh mass flux measurements in a free jet by imaging scattered light from a laser beam in the flow field directly through the etalon ${ }^{8}$. A similar technique has also been applied in the Rayleigh velocimetry work of Bivolaru et al. ${ }^{3}$

The original intent for the Rayleigh system developed in this work for use in the $15 \mathrm{~cm} \times 15 \mathrm{~cm}$ Supersonic Wind Tunnel (SWT) at NASA GRC, was to install a direct-imaging system like the one developed in Reference 8. The system was installed and tested in the wind tunnel, however the results were very poor due to extreme image distortion since the object plane and detection plane were at extreme angles to one another in addition to having an angled window in the collection path. Additionally, the weight of the equipment that had to be installed around the facility to implement such a system was very heavy and prohibited translation of the probe volume within the flow field. In the interest of limited tunnel testing time, the system was simplified to improve the signal levels and decrease the complexity of the data processing. Therefore, a fiber-coupled point-wise measurement approach was implemented to reduce the amount and weight of hardware needed around the facility test section. Image distortion was no longer an issue since this type of technique only required that light from the probe volume was collected into an optical fiber. The solid fused-silica FP etalon was used in close proximity to the wind tunnel to show that the facility noise and vibrations do not affect the data quality since the solid etalon is rigid. The interference pattern produced by the etalon was recorded using a low read-noise CCD camera. The measurements acquired in this work are done so in the presence of shocks. Shocks may have a significant effect on the laser propagation direction, which changes the component of the velocity that is measured and may also move the laser beam out of the depth of focus of the imaging system. The data will show that these aero-optical effects did not seem to be significant in this work. Measurements were acquired in an empty tunnel to establish the baseline flow characteristics in the tunnel and also in a supersonic combustions ramjet (scramjet) engine isolator model. A dynamic isolator decouples instabilities and disturbances from propagating between the inlet and supersonic combustor in a scramjet engine. Rayleigh scattering measurement capabilities in the isolator model will help researchers to understand the flow field and aid in using the isolator for flow control.

\section{Rayleigh Scattering and Fabry-Perot Interferometry}

In molecular Rayleigh scattering an incident electric field interacts with an atom or a molecule inducing a dipole moment that oscillates and radiates at the frequency of the incident field. It is considered an elastic scattering process because the internal energy of the molecule is unchanged and the frequency of the light is changed only by the Doppler effect due to the thermal as well as the bulk motion of the molecules ${ }^{1}$. The frequency spectrum of the scattered light contains information about the gas density, bulk velocity, and temperature. Figure 1 shows a Rayleigh scattering spectrum containing the narrow laser line and a typical Rayleigh spectral peak to illustrate how the flow property measurements are obtained from the spectral information. If the gas composition is fixed, the total intensity of the Rayleigh scattered light is directly proportional to the gas density $\rho$. The frequency shift between the laser peak and the Rayleigh peak is proportional to the bulk flow velocity $v_{k}$. The width of the spectrum is related to the gas temperature $T$. The measured velocity component, $v_{k}$, is in the same direction as the interaction wave vector $\mathbf{K}$, which is the bisector of the incident and scattered light wave vectors, $\mathbf{k}_{\mathbf{0}}$ and $\mathbf{k}_{\mathbf{s}}$, respectively, as shown in Fig. 2. The interaction wave vector and its magnitude $K$ are given by:

$$
\begin{gathered}
\mathbf{K}=\mathbf{k}_{\mathbf{s}}-\mathbf{k}_{\mathbf{0}} \\
K=|\mathbf{K}|=\frac{4 \pi}{\lambda}\left[\sin \frac{\chi_{\mathrm{s}}}{2}\right]
\end{gathered}
$$

The geometry of the optical arrangement in a particular experiment determines the component of the velocity vector $\mathbf{v}$ that is measured:

$$
v_{k}=\frac{\mathbf{K} \cdot \mathbf{v}}{K}
$$




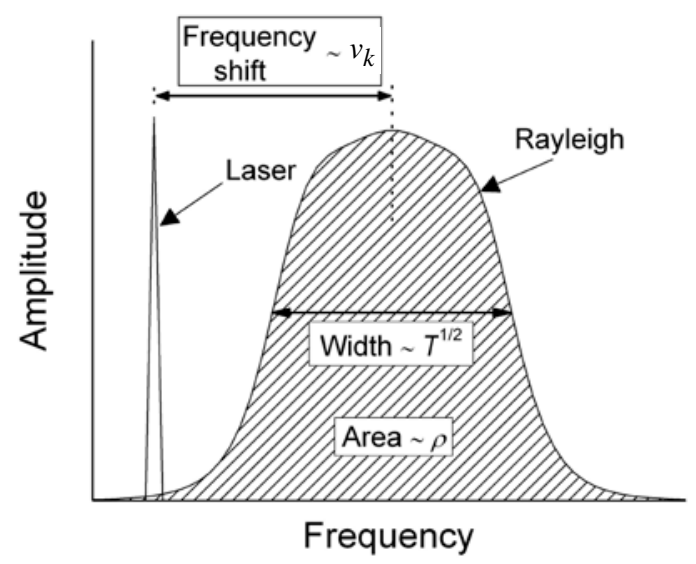

Fig. 1 Rayleigh scattering spectrum.

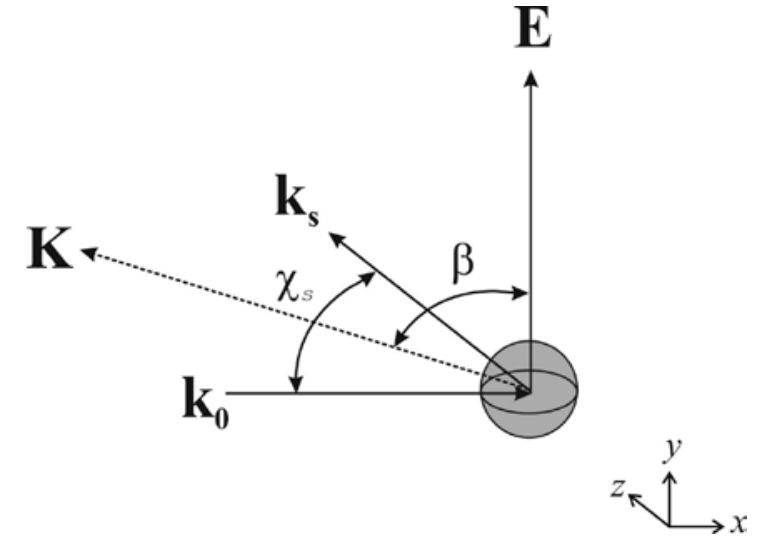

Fig. 2 Light scattering from a moving particle.

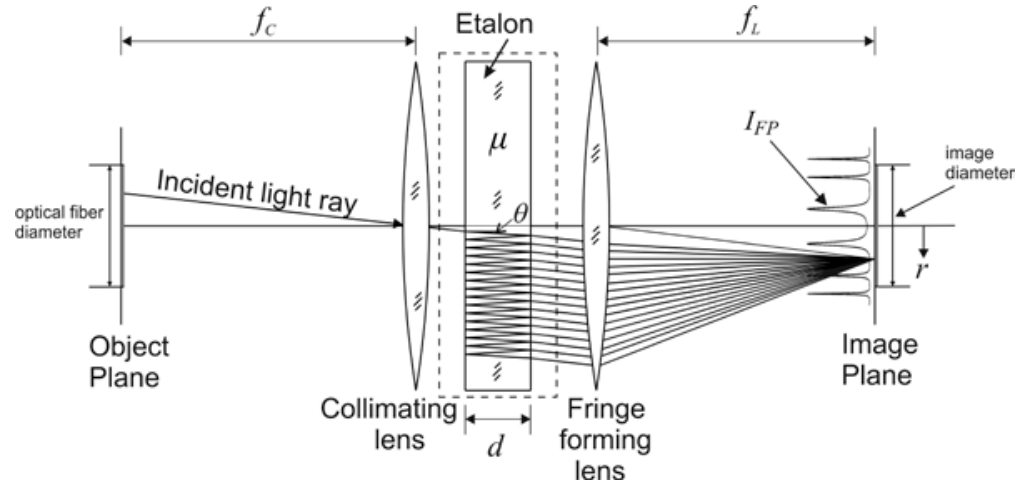

Fig. 3 Basic optical arrangement for spectrally-analyzing a uniform light source using a solid Fabry-Perot etalon.

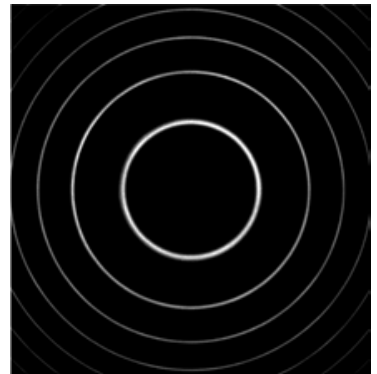

Fig. 4 Concentric ring interference pattern from a planar singlefrequency light source imaged through a Fabry-Perot etalon.

The spectrum of the Rayleigh scattered light is analyzed by imaging the scattered light through a planar solid FP etalon (Fig. 3) ${ }^{2}$. A detailed discussion of the theory of Fabry-Perot interferometry as applied to this type of Rayleigh scattering experiment can be found in Ref. 8. Figure 4 shows a typical fringe pattern that results when a planar single-frequency light source is imaged through a Fabry-Perot etalon. For spectrally broadened light, such as Rayleigh scattered light, the fringes broaden accordingly; the linewidth of the fringes provides a measure of temperature. The frequency shift of the light, which is associated with the bulk flow velocity, is determined by a spatial shift in the fringe positions in the image.

In this experiment, Rayleigh scattered light from a defined probe volume is collected into a multimode optical fiber. Some stray laser light scattered from the surrounding surfaces is also collected and imaged with the Rayleigh scattered light. At some tunnel setpoints, the flow contains water condensation which has a much larger scattering cross-section than the air molecules and dominates the Rayleigh scattering signal. The frequency of the Mie scattered light from the condensation is Doppler shifted just like Rayleigh scattered light. However, the condensation particles are too large to exhibit thermal fluctuations so the spectrum is not thermally-broadened like the Rayleigh spectrum. The incident laser beam with power $P_{0}$ is focused to a diameter smaller than the imaged field size, such that the power collected is proportional to the length of the probe volume set by the field size of the collection optics. The Rayleigh scattered light integrated over the collection solid angle $\Omega$ from a probe volume of length $L$ expressed in terms of expected photoelectron counts is: 


$$
\left\langle N_{R}\right\rangle=\int_{\Delta \Omega} \frac{\varepsilon P_{0} n L \lambda \Delta t \frac{\partial \sigma}{\partial \Omega} \sin ^{2} \beta}{h c} \partial \Omega
$$

where $\varepsilon$ is the overall system efficiency including detector quantum efficiency and other losses. With the interferometer in the optical path, the amount of energy, in terms of CCD grey level counts, collected on the $q^{\text {th }}$ pixel of the detector centered at position $\left(x_{q}, y_{q}\right)$ including light scattered from gas molecules (Rayleigh scattering), stray scattered light at the laser frequency, light scattered from condensation particles, broadband background light, and camera read noise is expressed as:

$$
\begin{aligned}
\left\langle N_{q}\right\rangle= & \frac{A_{R}}{\varepsilon_{p}^{2}} \int_{y_{q}-\frac{\varepsilon_{p}}{2}}^{y_{q}+\frac{\varepsilon_{p}}{2}} \int_{x_{q}-\frac{\varepsilon_{p}}{2}}^{x_{q}+\frac{\varepsilon_{p}}{2}} \int_{-\infty}^{+\infty} S_{R}(v) I_{F P}(v, x, y) d v d x d y \\
& +\frac{A_{f}}{\varepsilon_{p}^{2}} \int_{y_{q}-\frac{\varepsilon_{p}}{2}}^{y_{q}+\frac{\varepsilon_{p}}{2}} \int_{x_{q}-\frac{\varepsilon_{p}}{2}}^{x_{q}+\frac{\varepsilon_{p}}{2}} I_{F P}\left(v_{0}, x, y\right) d x d y \\
+ & \frac{A_{p}}{\varepsilon_{p}^{2}} \int_{y_{q}-\frac{\varepsilon_{p}}{2}}^{y_{q}+\frac{\varepsilon_{p}}{2}} \int_{x_{q}-\frac{\varepsilon_{p}}{2}}^{x_{q}+\frac{\varepsilon_{p}}{2}} I_{F P}(v, x, y) d x d y+A_{b}
\end{aligned}
$$

where the amplitude of the Rayleigh scattered light is defined as:

$$
A_{R}=\left(\left\langle N_{R}\right\rangle \varepsilon_{p}^{2}\right) /\left(G \pi r_{\max }^{2}\right)
$$

The second and third terms in Eq. (5) are associated with scattered light at the laser frequency and scattered light from particles (Mie scattering), respectively. The amplitude of broadband background light plus the camera read noise is represented by the constant $A_{b}$; this value is dominated by camera read noise since the level of broadband light in the facility is minimized during testing. The Rayleigh spectrum $S_{R}$ is evaluated using the TENTI S6 kinetic theory model ${ }^{9,10}$. The imaged fringe patterns are analyzed by maximum likelihood estimation (MLE) ${ }^{11}$ analysis using the model function described in Eq. (5) to extract the density and velocity information.

An image representative of data containing Rayleigh signal and reference laser signal acquired in the $15 \times 15$ SWT is shown in Fig. 5. An image that contains reference laser signal and Mie scattering from condensation in the engine isolator model test in the 15x15 SWT is shown in Fig. 6. Velocity measurements were obtained in both cases; however density measurements were only possible in situations such as shown in Fig. 5 where Mie scattering was not present. The amplitude of the particle scattering was typically an order of magnitude greater than the Rayleigh scattering making it impossible to recover any information contained in the Rayleigh signal. The condensation particles were probably on the order of $5-10 \mu \mathrm{m}$ in size with Mie scattering cross sections about 20 orders of magnitude greater than the Rayleigh scattering cross sections. The number density of the condensation particles was assumed to be quite low since the Mie scattering signals are only an order of magnitude greater than the Rayleigh signals. 


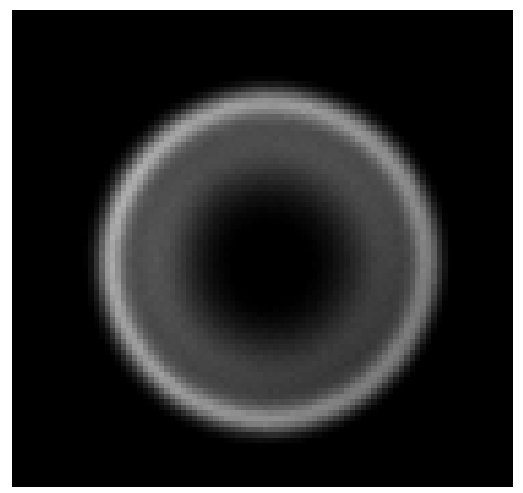

Fig. 5 Rayleigh light and reference laser light acquired in a Mach 3 flow $(\mathrm{Re}=7 \mathrm{E} 6 / \mathrm{ft})$ in the 15x15 SWT test section and imaged through a solid FP etalon. The Rayleigh signal is contained in the dimmer inner ring of the concentric interference pattern. Camera integration time was 5 seconds.

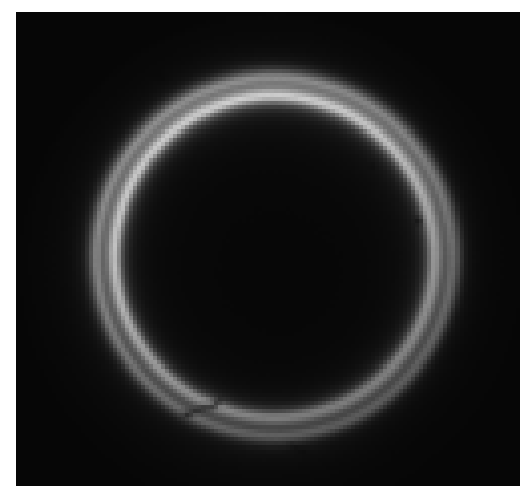

Fig. 6 Mie scattered light and reference laser light collected from a Mach 3 flow $(\operatorname{Re}=7 E 6 / f t)$ in an engine isolator model in the 15x15 SWT and imaged through a solid FP etalon. The Mie signal is contained in the brighter inner ring of the concentric interference pattern. Camera integration time was 4 seconds.

\section{Experiment}

\section{A. Experimental setup}

Figure 7 shows a top-view diagram of the Rayleigh measurement system arrangement in the 15x15 SWT. Rayleigh measurements were acquired in two different wind tunnel configurations. The first test was performed with an empty test section (i.e., no model present) to evaluate the baseline tunnel flow characteristics. In the second test, velocity measurements were acquired in the flow through an engine isolator model that was installed in the test section of the wind tunnel. A dynamic isolator is necessary between the inlet and combustor of scramjet engines to isolate instabilities and disturbances from propagating either upstream or downstream, decoupling the inlet and combustor flows. A side-view schematic of the tunnel with the isolator model installed is shown in Fig. 8 where the model components are shown in blue. The tunnel was operated at a Mach number of 3.0 in both test configurations. The tunnel total pressure was varied to operate the tunnel at three different Reynolds numbers. The adjustable isolator parameters were the angle of the top plate, and the axial position of the wedge near the isolator exit. These model parameters were adjusted to provide clean isolator started flow conditions as well as a flow with an oblique shock train. Schlieren images of the clean and oblique shock train flow conditions are shown in Figs. 9(a) and 9(b), respectively.

A Coherent Verdi $10 \mathrm{~W}$ continuous-wave 532-nm wavelength Nd:Vanadate laser with a 2.25-mm diameter output beam and $5 \mathrm{MHz}$ linewidth provided the incident light for the system. The vertically-polarized laser beam was focused with a $300-\mathrm{mm}$ focal length lens (Lens 1) to a $90-\mu \mathrm{m} 1 / \mathrm{e}^{2}$ diameter at the probe volume. The beam propagated orthogonal to the primary flow direction (y-direction) and light was collected at a $45^{\circ}$ angle from forward scattering. The incident and scattering wave vectors resulted in a measured velocity component along the direction indicated by the blue $\mathbf{K}$ vector in Fig. 7. Although the velocity vector is not completely in the axial $(x)$ direction, the flow component in the $y$-direction is assumed to be approximately zero in order to estimate the axial velocity. A finite length of the laser beam was imaged by a pair of f/6 300-mm achromatic lenses (Lenses 2 and 3) onto the face of a 0.91-mm multimode optical fiber. Since the lenses provided one-to-one imaging, the probe volume length was 0.91-mm orthogonal to the primary flow direction and the probe volume width, which was set by the focused beam waist, was $0.09-\mathrm{mm}$. The probe volume was located approximately $16 \mathrm{~cm}$ downstream of the leading edge of the test section window and $5.5 \mathrm{~cm}$ above the tunnel floor in the baseline (empty test section) study. The isolator model top plate, which was $50 \mathrm{~cm}$ long and mounted parallel to the tunnel floor at a height of $5.1 \mathrm{~cm}$, is shown in blue in Figs. 7 and 8 to illustrate the location of model relative to the optics and probe volume. The probe volume was approximately $36 \mathrm{~cm}$ downstream of the leading edge of the isolator plate and $3.9 \mathrm{~cm}$ from the tunnel floor in the isolator model test. Breadboard 2 was mounted below the wind tunnel test section on a traverse that had a travel range of $10 \mathrm{~cm}$. Translation of the optics on this breadboard enabled the probe volume to be translated orthogonal to 
the primary flow direction from the tunnel wall to a position about $2.5 \mathrm{~cm}$ past the tunnel centerline. Data was not acquired any closer than about $2 \mathrm{~cm}$ from the tunnel wall since the amplitude of the stray laser light reflecting from the test section window became too prohibitive for practical measurements. There were numerous physical limitations that prohibited axial translation of the probe volume in the wind tunnel. The schematic shows that the collection cone of Lens 2 is barely clearing the window leading edge. If the probe volume were translated upstream of its current location the collection cone would be clipped, reducing the total collected signal. There were also physical constraints imposed by the tunnel hardware that are not depicted in the schematic.

The 20-m long, 0.91-mm diameter multimode optical fiber transmitted the collected signal, which included the Rayleigh signal, stray laser light, and in some cases, Mie signal from condensation particles, to breadboard 3 which was located in close proximity to the wind tunnel and contained the FP etalon and CCD camera for spectral analysis of the light. The light exiting the fiber was collimated by a 60 -mm focal length $f / 2.4$ lens (Lens 4 ) and was directed through the solid FP etalon. The 25-mm diameter etalon was made of fused silica with a refractive index of 1.460711 at room temperature. The etalon had $90 \%$ reflectivity coatings providing a reflective finesse of 30 . The etalon was approximately $11.3-\mathrm{mm}$ thick giving a free spectral range of $9 \mathrm{GHz}$. The light exiting the etalon was focused by a Nikon f/4 200-mm focal length lens at the detector of a Princeton Instruments VersArray backilluminated, scientific-grade CCD camera. The $512 \times 512$ imaging array had a $100 \%$ fill factor, $24 \times 24-\mu \mathrm{m}$ pixels, 16 bit dynamic range, high quantum efficiency, thermoelectric cooling and low-noise electronics. The image of the optical fiber face was approximately 125 pixels in diameter on the CCD detector. Images were acquired with varying exposure times depending on the signal levels from the various scattering contributions. A typical exposure time was 5 seconds.

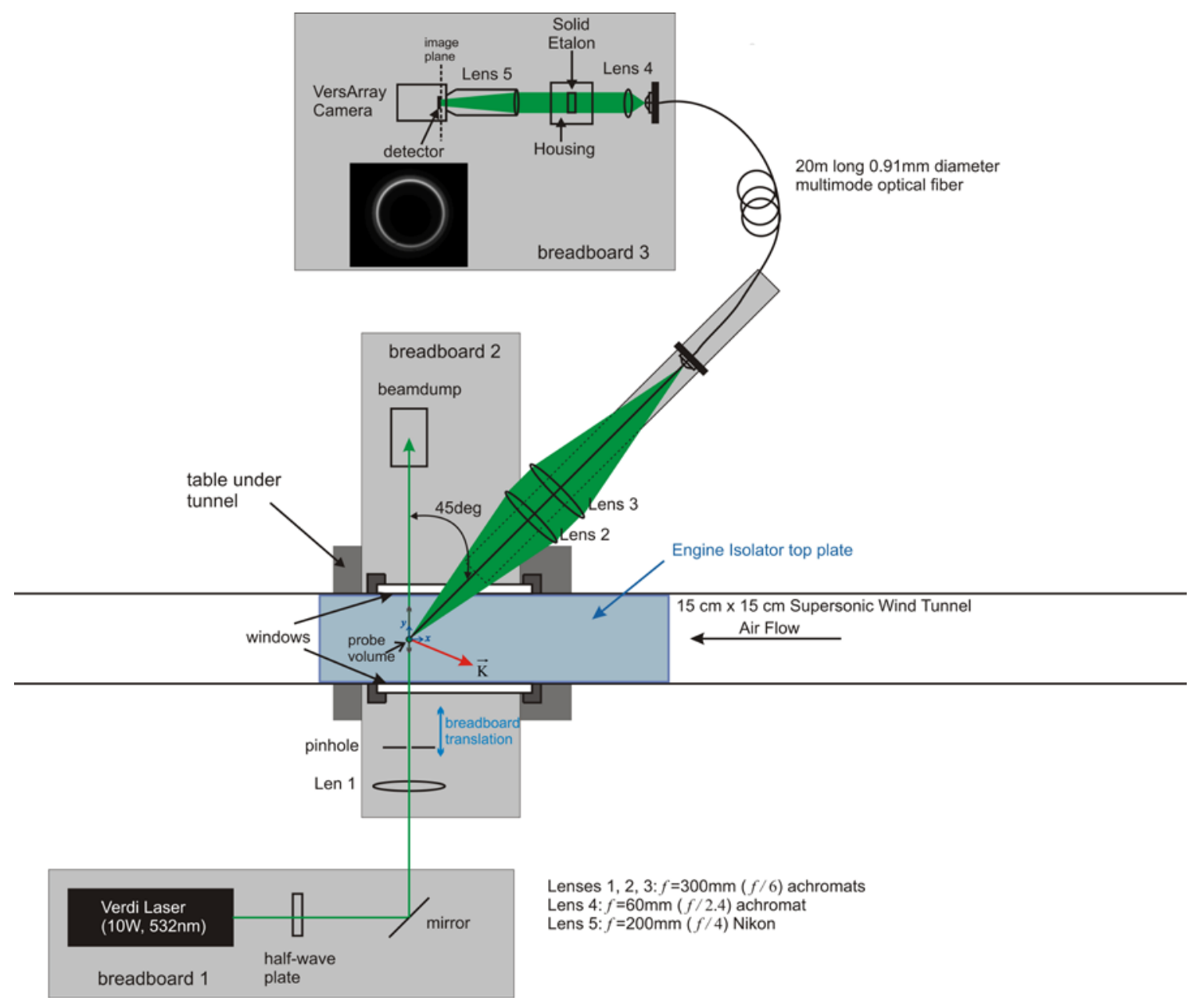

Fig. 7 Schematic of Rayleigh mass flux measurement system in the 15x15 SWT (top view). 


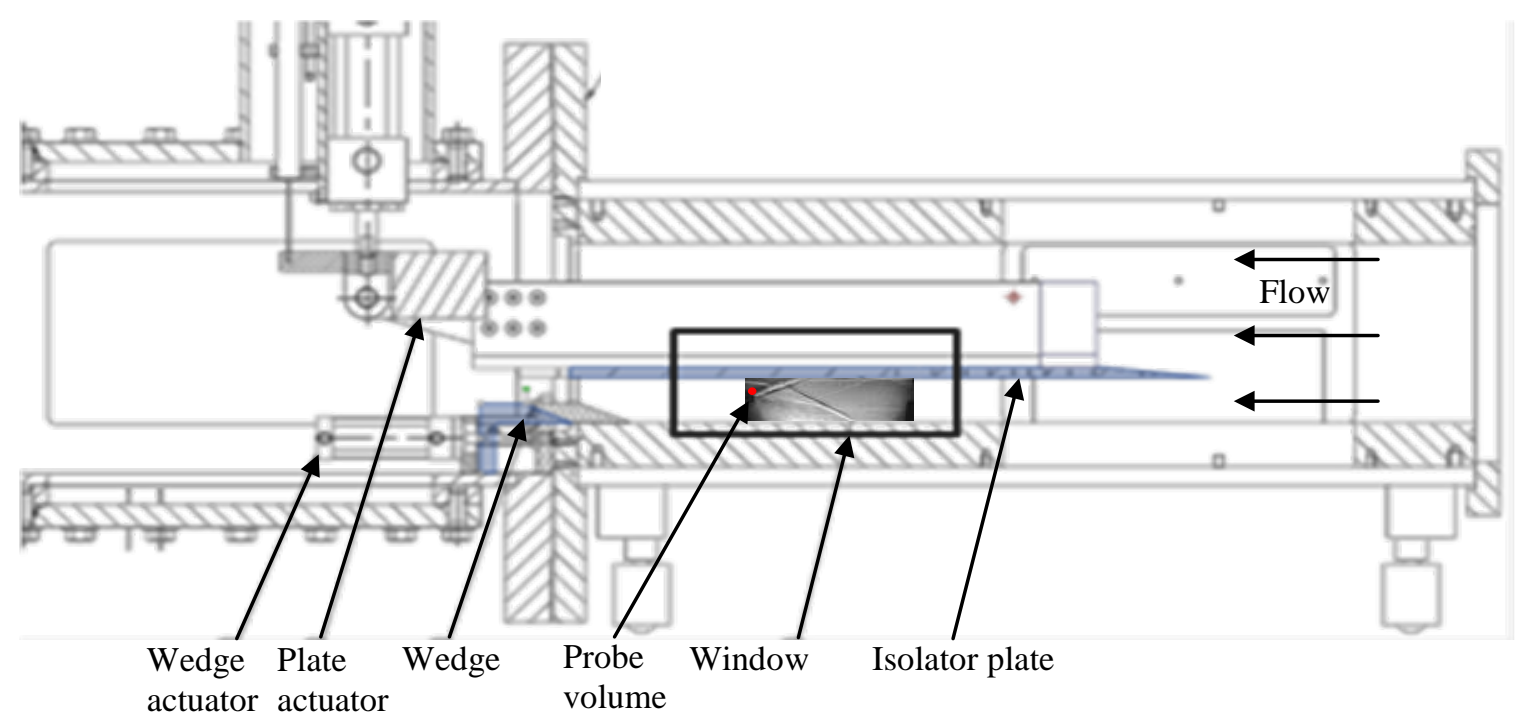

Fig. 8 Schematic of the isolator model (components shown in blue) in the 15x15 SWT (side view). The bold black rectangle indicates the window port used for the Rayleigh system and the red circle indicates the probe volume location. A Schlieren image of a flow with an oblique shock train acquired with this tunnel and model configuration is overlaid on the schematic.
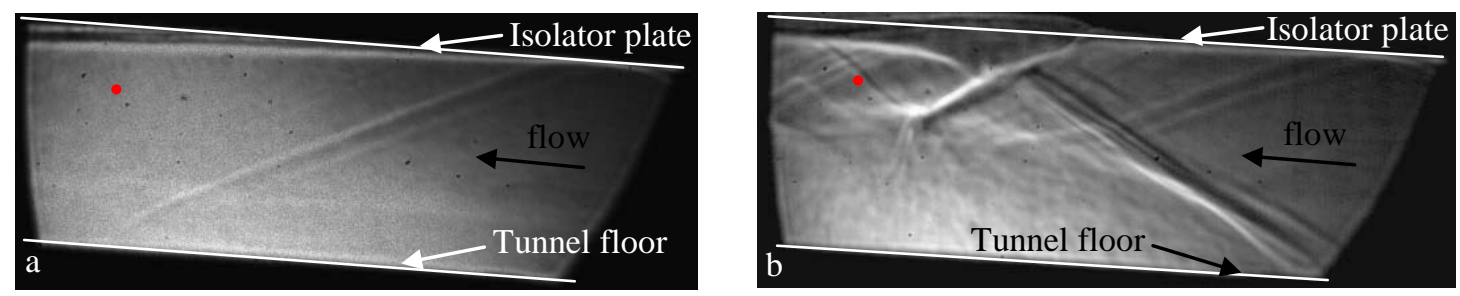

Fig. 9 Schlieren image data in the engine isolator model. Flow is left to right. The image on the left is representative of a clean started flow case (a) while the image on the right is representative of the oblique shock train case (b). The probe volume was $3.9 \mathrm{~cm}$ from the tunnel floor at roughly the location indicated by the red circle in each image.

\section{B. Data analysis method}

The only system parameter that needed to be calibrated was the optical system efficiency $\varepsilon$. The static and stagnation pressures measured by pressure transducers in the tunnel and the stagnation temperature measured by a thermocouple in the tunnel plenum were used in the isentropic flow relations to estimate the static density in the tunnel. Several images acquired near the centerline of the tunnel at Reynolds numbers of 3E6/ft, 5E6/ft, and 7E6/ft were analyzed to calibrate the efficiency factor. The efficiency factor was estimated from MLE using the model function given in Eq. (5) and holding the tunnel density fixed at the isentropic value. After calibration, MLE analysis using the same model function was used to provide the desired parameter estimates. In the cases where only Rayleigh and reference signal were present, the image data were fit for $\rho$ and $v_{k}$ from the Rayleigh signal, and $A_{f}$ and the reference fringe radius from the reference signal, as well as the center of the circular fringe pattern $\left(x_{0}, y_{0}\right)$ and the broadband light amplitude $A_{b}$. Since the optical arrangement provided a measured velocity component that was at an angle of $22.5^{\circ}$ from the axial direction, the velocity in the y-direction was assumed to be zero and the axial velocity component was calculated by the following equation: 


$$
u=\frac{v_{k}}{\cos \left(22.5^{\circ}\right)}
$$

The simultaneous density and axial velocity measurements were then used to calculate mass flux $(=\rho u)$. Although temperature estimates were not of interest in this work, the gas temperature affects the width and shape of the spectrum so it was necessary to provide a reasonable estimate of the temperature in the model function. Therefore, the temperature was estimated from the tunnel static pressure and the gas density estimated from the fringe data using the Ideal Gas Law. In the cases where Mie scattering from water condensation overwhelmed the Rayleigh signal, the image data were fit for $v_{k}$ from the Mie signal, and $A_{f}$ and the reference fringe radius from the reference signal, as well as the center of the circular fringe pattern $\left(x_{0}, y_{0}\right)$ and the broadband light amplitude $A_{b}$. As above, Eq. (7) was used to calculate the axial velocity from the measured velocity component.

Figure 10 shows a profile of the interference fringe data (circular symbols) and the corresponding model function (solid line) fit to the data from the baseline test (no model in test section) at a Reynolds number of 7E6/ft in the $15 \times 15$ SWT. This data corresponds to the image that was presented in Fig. 5. The reference light is the sharp peak and the Rayleigh signal is the broader peak. Because the $\mathbf{K}$ vector is oriented in the negative direction to the main flow direction the Rayleigh fringe is shifted more toward the center of the fringe pattern than the reference laser peak. The measured and theoretical velocity and density are indicated in the figure caption. Figure 11 shows a similar profile for data corresponding to the image that was presented in Fig. 6 that was acquired in the engine isolator model test at a Reynolds number of 7E6/ft. Both the reference laser peak and the Doppler shifted Mie scattering peak are approximately the same width since larger particles like the water condensation does not exhibit thermal motion and hence does not produce thermal broadening in the spectrum like Rayleigh signals. The measured velocity is indicated in the figure caption.

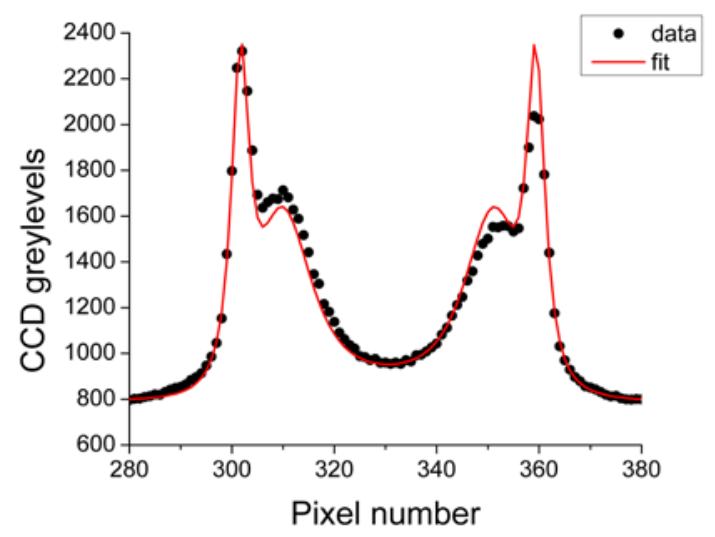

Fig. 10 Horizontal profile through the center of the fringe image data and corresponding model function fit to the Rayleigh and reference signals that were presented in Fig. 5. The measured velocity and density were $619.3 \mathrm{~m} / \mathrm{s}$ and $0.251 \mathrm{~kg} / \mathrm{m}^{3}$ and the expected velocity and density were $619.0 \mathrm{~m} / \mathrm{s}$ and $0.264 \mathrm{~kg} / \mathrm{m}^{3}$,

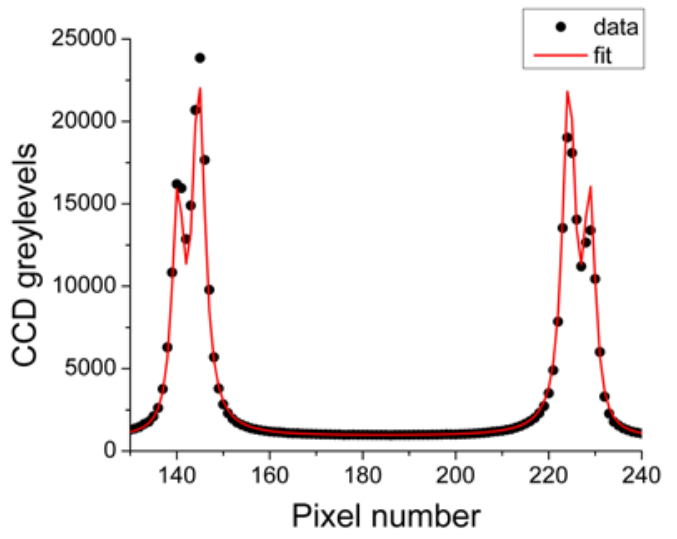

Fig. 11 Horizontal profile through the center of the fringe image data and corresponding model function fit to the Mie and reference signals that were presented in Fig. 6. The measured velocity was $548 \mathrm{~m} / \mathrm{s}$. The true velocity is unknown but it should be less than the isentropic velocity, which was $623 \mathrm{~m} / \mathrm{s}$, due to the presence of the oblique shock train. The density could not be determined with Mie scattering present. 


\section{Results and Recommendations}

\section{A. Baseline Tunnel Flow}

Velocity and density measurements were acquired in the test section of the 15x15 SWT operated at Mach 3 and Reynolds numbers of 3E6/ft, 5E6/ft, and 7E6/ft to validate the Rayleigh technique and characterize the baseline wind tunnel flow when no model was present in the test section. The probe volume was translated across the tunnel orthogonally to the axial flow direction over the range of $y=-5.6 \mathrm{~cm}$ to $y=+2.4 \mathrm{~cm}$, where $y=0$ is the tunnel centerline and $y= \pm 7.5 \mathrm{~cm}$ are the tunnel sidewalls. The probe volume was only translated from $y=-5.6 \mathrm{~cm}$ to $y=0$ at the $\mathrm{Re}=3 \mathrm{E} 6 / \mathrm{ft}$ condition. Measurements were not acquired within $2 \mathrm{~cm}$ of the tunnel side walls because the stray laser signal was too intense at those locations due to strong reflections from the windows. The axial velocity results for the three Reynolds numbers are presented in Fig. 12. The theoretical velocity is indicated in the figure. The measured velocities closer to the left tunnel side wall were within $10 \mathrm{~m} / \mathrm{s}$ of the theoretical values but the accuracy of the measurement appears to decline as the probe volume approaches the tunnel centerline. The reduced accuracy is directly related to an increase in reference laser signal in the images.

Figure 13 shows several fringe profiles from this data set at various $y$ locations showing that the Rayleigh peak is very hard to distinguish in the cases where the laser amplitude is very high. The velocity uncertainty was estimated from a Camer-Rao lower bound uncertainty analysis ${ }^{12}$ for all data points and the results are plotted in Fig. 14 along with the measured laser signal amplitude as a function of $y$ location in the tunnel. The laser signal varies as the probe volume is translated through the tunnel. The laser signal is greatest at $y=0$ where the collection optics are pointed at a region on the opposite tunnel wall where there is a lot of scattered laser light (the tunnel walls were not painted or treated to minimize flare light). The Rayleigh signal level is directly related to the molecular number density; the density is lowest in the $\mathrm{Re}=3 \mathrm{E} 6 / \mathrm{ft}$ case (velocity is constant at each Reynolds number). The uncertainty levels are the highest when the ratio of Rayleigh signal to laser signal is the lowest. Therefore the highest uncertainty levels occur in the low Reynolds number case at the tunnel centerline where the laser signal is nearly 10 times greater than the Rayleigh signal. This is definitely apparent in the velocity data in Fig. 12 since the velocity estimates at $\operatorname{Re}=$ 3E6/ft and $y=0$ are more than $100 \mathrm{~m} / \mathrm{s}$ below the expected values. This shows how critical the relative signal levels are to obtaining accurate flow measurements in this technique. Similar issues have been encountered by Bivolaru et al. ${ }^{3}$ in their use of Rayleigh scattering in various test facilities. They have implemented a method of obtaining a "flow off" reference image that is subtracted from their "flow on" image to help mitigate this problem. A similar technique will be tested in future Rayleigh experiments.

The static density measurements for the three Reynolds numbers are presented in Fig. 15 and the theoretical density values for each case are indicated in the figure. The measurements have accuracies of $4 \%$ or better compared to the expected values. The measured density and velocity values were used to calculate the mass flux at each measurement location and the results are presented in Fig. 16 along with the theoretical mass flux values for each Reynolds number case.

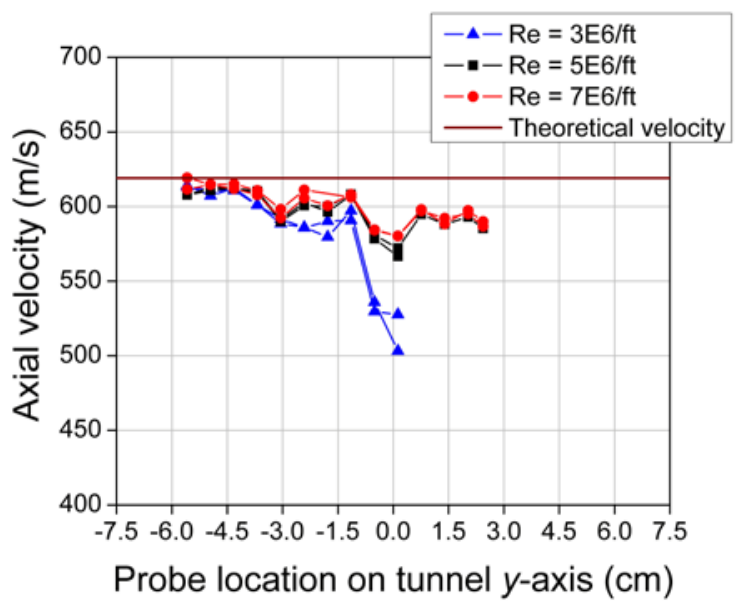

Fig. 12 Axial velocity measurements acquired in the 15x15 SWT at three Reynolds numbers (baseline test, no model present in test section).

10

American Institute of Aeronautics and Astronautics 


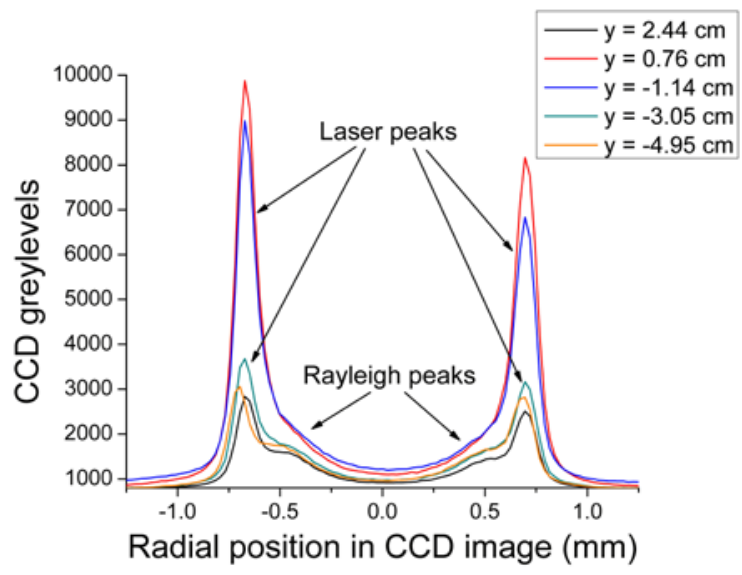

Fig. 13 Fringe image profiles at various $y$-locations in the tunnel demonstrating the varying amplitudes of laser signal compared to the low amplitude Rayleigh signals. When the laser amplitude is much greater than the Rayleigh amplitude the measurement uncertainty is high.

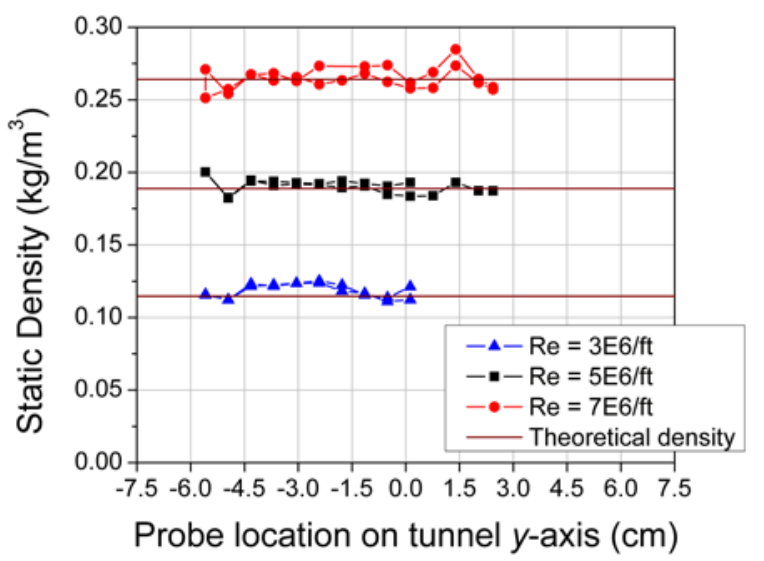

Fig. 15 Static density measurements acquired in the 15x15 SWT at three Reynolds numbers (baseline test).

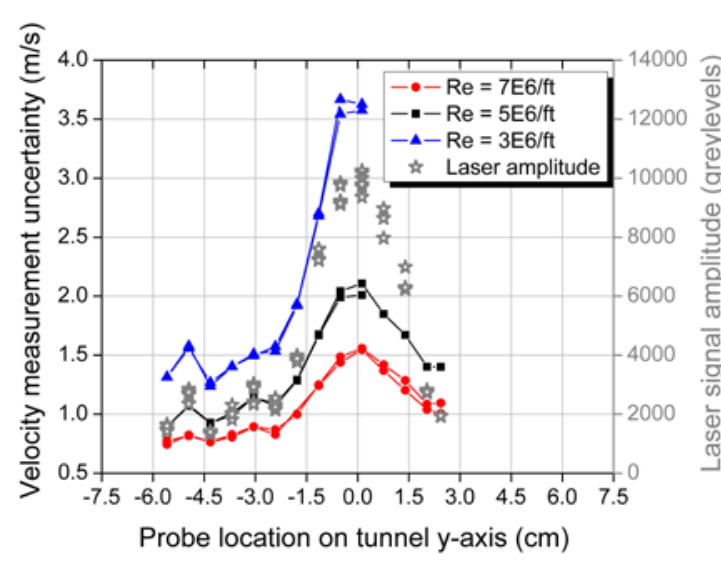

Fig. 14 Velocity measurement uncertainty and laser signal amplitude as a function of $y$ location in the tunnel for measurements acquired in the 15x15 SWT at three Reynolds numbers (baseline test).

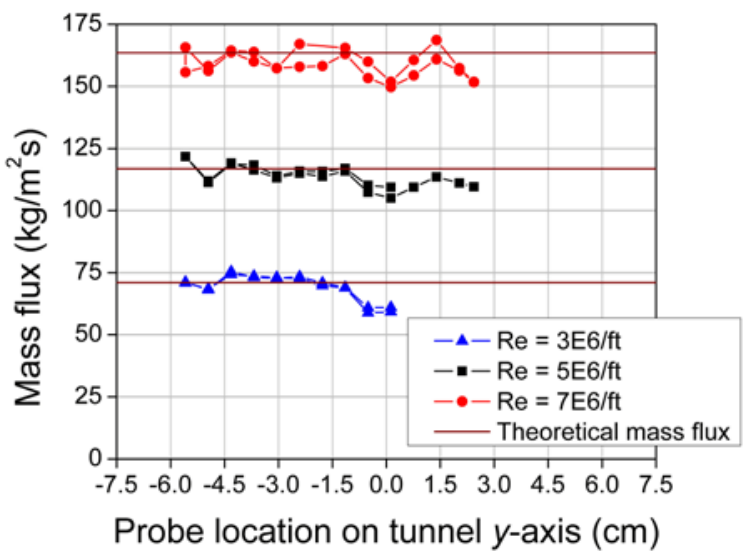

Fig. 16 Mass flux calculated from velocity and density measurements acquired in the 15x15 SWT at three Reynolds numbers (baseline test).

\section{B. Engine Isolator Testing}

The Rayleigh measurement system was also used to acquire flow measurements within an engine isolator model installed in the tunnel test section. The tunnel was again operated at Mach 3 and Reynolds numbers of 3E6/ft, 5E6/ft, and 7E6/ft. The isolator plate angle and wedge location were adjusted to provide two different operating points at each Reynolds number; a started "clean" flow case and a case with an oblique shock train present within the isolator. Schlieren images of the two flow conditions were shown in Fig. 9. Some weak shocks were also present in the clean flow case. In most cases measurements were acquired at only two $y$ locations: $y=-2.4 \mathrm{~cm}$ and $y=0.1$ $\mathrm{cm}$; however a profile from $y=-4.3 \mathrm{~cm}$ to $y=+1.4 \mathrm{~cm}$ was obtained for the shock train case at $\mathrm{Re}=5 \mathrm{E} 6 / \mathrm{ft}$. The flow at all test conditions was plagued by water condensation during this test possibly due to high humidity levels that day. Therefore the Rayleigh signal was corrupted by Mie scattering from the condensation. The Mie scattering corrupted signal provides a sharp Doppler shifted peak for estimating the velocity. Density measurements were not possible, however. The axial velocity results for the clean flow case are presented in Fig. 17. The theoretical velocity 
is indicated in the figure. The measured values for the higher Reynolds number cases are within $\pm 4 \mathrm{~m} / \mathrm{s}$ whereas the measured values for the $\mathrm{Re}=3 \mathrm{E} 6 / \mathrm{ft}$ case are $8-17 \mathrm{~m} / \mathrm{s}$ higher than expected. The Mie scattering signal level was about 10 times lower than the laser signal in the $\mathrm{Re}=3 \mathrm{E} 6 / \mathrm{ft}$ case which most likely is the reason for the reduction in accuracy for this case. As observed in the previous study of the baseline tunnel flow, it is critical to have the right balance between reference and Rayleigh scattering signals to achieve a minimum uncertainty level.

The axial velocity results for the case where the oblique shock train was present within the isolator are shown in Fig. 18. The theoretical velocity upstream of the shock is indicated in the figure. The velocities downstream of the shock are expected to be lower although it is unknown exactly what the expected velocity should be. The mass flow should be constant and therefore should be the same as in the clean flow case; however without valid density measurements this could not be verified. The $\mathrm{Re}=3 \mathrm{E} 6 / \mathrm{ft}$ case for the shock train flow condition also had very low Mie scattering signals resulting in possibly inaccurate velocity estimates. From the Re $=5 \mathrm{E} 6 / \mathrm{ft}$ and $\mathrm{Re}=7 \mathrm{E} 6 / \mathrm{ft}$ cases it appears that the flow velocity in the tunnel is about $555 \mathrm{~m} / \mathrm{s}$ behind the oblique shock train. The velocities measured in the $\mathrm{Re}=3 \mathrm{E} 6 / \mathrm{ft}$ case seem to be about $20-30 \mathrm{~m} / \mathrm{s}$ higher than expected.

One of the things we were interested in studying in these tests was the effect of shocks on the measurement technique. There were plenty of shocks throughout these flow fields but we did not observe any major signal loss due to beam steering issues related to laser-shock interactions. The Rayleigh signals were quite low in these tests so a goal for future testing is to enhance the detected Rayleigh signals. Another goal is to reduce excessive stray laser light from the tunnel walls and windows to increase measurement accuracy and to enable measurements closer than $2 \mathrm{~cm}$ from the tunnel wall.

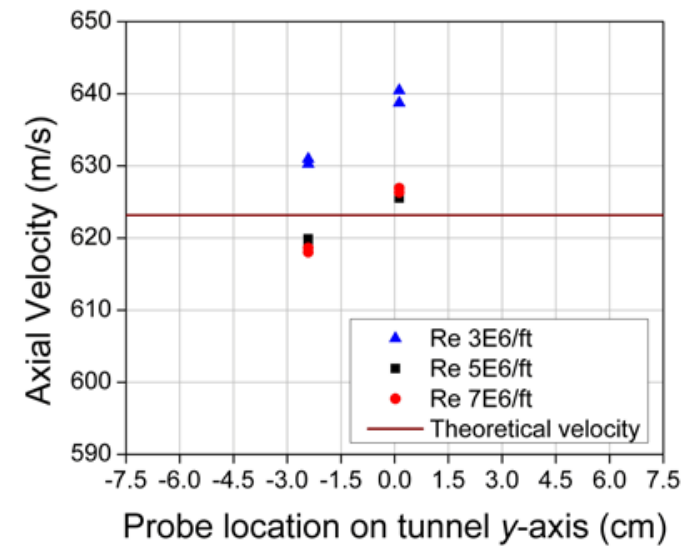

Fig. 17 Axial velocity measurements acquired in the engine isolator model in the 15x15 SWT at three Reynolds numbers with clean flow conditions.

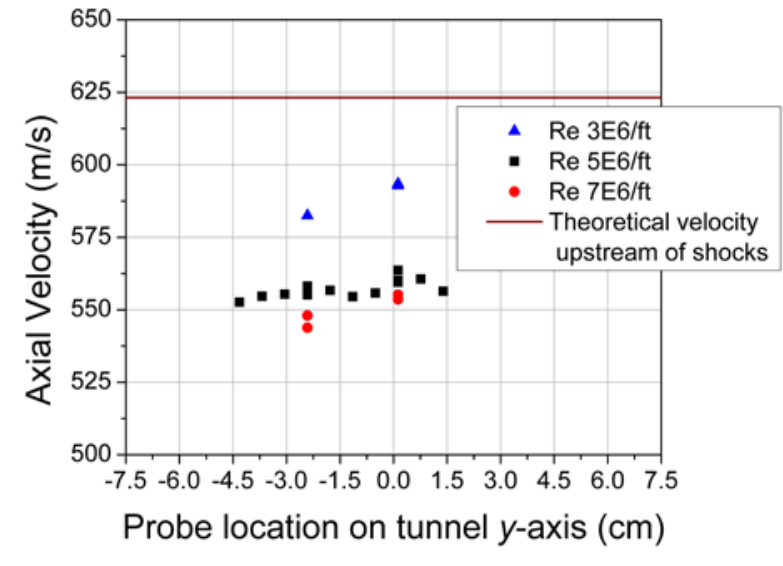

Fig. 18 Axial velocity measurements
acquired in the engine isolator model in the
15x15 SWT at three Reynolds numbers with
oblique shock train flow conditions.

\section{Conclusions and Future Work}

A Rayleigh scattering mass flux measurement technique was developed in which Rayleigh scattered light and reference laser light were collected by an optical fiber and transmitted to another location for spectral analysis and detection using a solid etalon and a low-read-noise CCD camera. This system was installed in the $15 \mathrm{~cm}$ x15 cm Supersonic Wind Tunnel at NASA Glenn Research Center and data were acquired in an empty tunnel (baseline flow case) and in an engine isolator model at Mach 3.0 tunnel conditions and three different Reynolds numbers. The probe volume was translated across the tunnel orthogonal to the primary flow direction to map out a linear region of the flow field. The presence of shocks in the flow did not appear to have any detrimental effects on the measurements. Stray light from the tunnel walls and other surfaces was not strong enough to degrade the Rayleigh data in most cases; however a goal for future testing is to reduce stray light levels to maintain a more desirable ratio between Rayleigh and laser signals to minimize uncertainty. The presence of condensation in the flow during the engine isolator tests prohibited density measurements. The test may be repeated in the future to see if running on a day with a lower humidity level reduces the condensation levels. 


\section{Acknowledgments}

The authors would like to thank John Dearmon, Gerry Buchar, and Scott Smrdel for providing facility test support during the tunnel operation. This work was supported by the Hypersonics discipline under NASA's Fundamental Aeronautics Program.

\section{References}

${ }^{1}$ Miles, R. B., Lempert, W. R., and Forkey, J. N., “Laser Rayleigh Scattering,” Meas. Sci. Technol., Vol. 12, No. 5, 2001, pp. R33-R51.

${ }^{2}$ Vaughan, J. M., The Fabry Perot Interferometer, History, Theory, Practice, and Applications, Adam Hilger, Philadelphia, 1989, pp. 89-134.

${ }^{3}$ Bivolaru, D., Cutler, A. D., Danehy, P. M., Gaffney, R. L., and Baurle, R. A., "Spatially and Temporally Resolved Measurements of Velocity in a $\mathrm{H}_{2}$-air Combustion-heated Supersonic Jet,” AIAA-2009-27, 2009.

${ }^{4}$ Seasholtz, R. G., Buggele, A. E., and Reeder, M. F., "Flow Measurements Based on Rayleigh Scattering and Fabry-Perot Interferometer," Optics and Lasers in Engineering, Vol. 27, No. 6, 1997, pp. 543-570.

${ }^{5}$ Panda, J., and Seasholtz, R. G., "Velocity and Temperature Measurement in Supersonic Free Jets Using Spectrally Resolved Rayleigh Scattering,” AIAA-99-0296, 1999.

${ }^{6}$ Seasholtz, R. G., Panda, J., and Elam, K. A., "Rayleigh Scattering Diagnostic for Measurement of Velocity and Density Fluctuation Spectra,” AIAA-2002-0827, 2002.

${ }^{7}$ Mielke, A. F., Elam, K. A., and Sung, C. J., “Time-Resolved Rayleigh Scattering Measurements in Hot Gas Flows,” AIAA2008-262, 2008.

${ }^{8}$ Mielke, A. F., Elam, K. A., Clem, M. M., "Multiple-Point Mass Flux Measurement System Using Rayleigh Scattering,” AIAA-2009-528, 2009.

${ }^{9}$ Tenti, G., Boley, C. D., and Desai, R. C., "On the Kinetic Model Description of Rayleigh-Brillouin Scattering from Molecular Gases,” Canadian Journal of Physics, Vol. 52, No. 4, 1974, pp. 285-290.

${ }^{10}$ Boley, C. D., Desai, R. C., and Tenti, G., "Kinetic models and Brillouin scattering in a molecular gas," Canadian Journal of Physics, Vol. 50, No. 18, 1972, pp. 2158-2173.

${ }^{11}$ Edwards, R. V., Processing Random Data: Statistics for Engineers and Scientists, World Scientific, New Jersey, 2006, pp.91-97.

${ }^{12}$ Whalen, A. D., Detection of Signals in Noise, Academic Press, New York, 1971, pp. 327-332. 